\title{
Quality Assurance (QA) for Kilovoltage Cone Beam Computed Tomography (CBCT)
}

\author{
Joerg Lehmann ${ }^{1,2}$ and Stanley Skubic ${ }^{2}$ \\ ${ }^{1}$ University of California Davis, Davis, CA, \\ ${ }^{2}$ Radiological Associates of Sacramento, Sacramento, CA, \\ USA
}

\section{Introduction}

The use of an imaging modality for verification of patient and target location immediately prior to application of radiation therapy has spread widely in the last years. It is referred to as Image Guided Radiation Therapy (IGRT).

Precise targeting of the tumor is very important and imaging can be immensely helpful in this process. Hence the quality assurance of the imaging systems used to guide the beam is also essential. One could argue that it is as critical as quality assurance for the radiation dose output, since the right amount of radiation dose delivered to the wrong location can be as detrimental as delivering the wrong amount of radiation dose to the right location.

Kilovoltage $(\mathrm{kV})$ Cone Beam Computed Tomography (CBCT) is a premiere modality for image guidance, since it produces three-dimensional images of the region of interest of the patient in a relatively short time (1-3 $\mathrm{min})$ in a sufficient quality for the purpose of patient alignment. Popular systems are the Varian OBI (Varian Medical Systems, Inc., Palo Alto, CA, USA) and the Elekta XVI system (Elekta AB, Stockholm, Sweden), shown in figure 1 .

This chapter introduces the different tests needed for a comprehensive QA of kV CBCT. The tests are related to (1) System safety, (2) Geometric accuracy, that is agreement of the CBCT imaging isocenter with the isocenter of the MV treatment beam, (3) Registration and correction accuracy, that is how accurately can the system position a patient, (4) Image quality, and (5) x-ray tube and generator performance (dose to the patient). It further suggests a frequency with which each of the tests should be performed, organizing them in being part of the daily, monthly and annual QA procedures. In addition, tests which should be performed following system repair or upgrades are described.

Responsibility for all tests should be with a Qualified Medical Physicist. Generally the physicist will delegate some of the tests, likely the daily tests to other members of the Radiation Therapy team, for instance the Radiation Therapists. (Klein et al., 2009).

For information about in-room $\mathrm{kV}$ X-ray imaging beyond CBCT QA see the report of AAPM Task Group 104 (Yin et al., 2009) and (Jaffrey et al., 1999). 

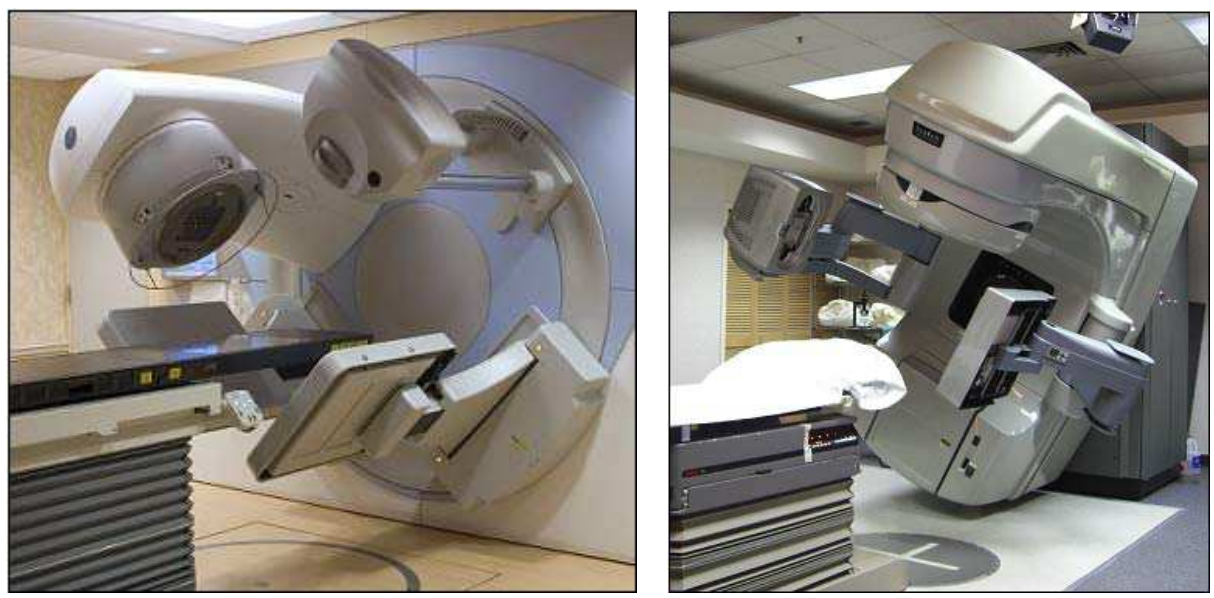

Fig. 1. Linear accelerators equipped with $\mathrm{kV}$ imaging systems capable of CBCT, left: Elekta Synergy ${ }^{\circledR}$ with XVI, right: Varian 21EX with OBI (all images by the author JL)

\section{2. СВCT test descriptions}

\subsection{System safety}

The system safety checks are concerned with gross safety issues, such as measures to avoid injury to the patient through collision with a remote controlled part of the system and access control to the room. Some of the tests will be very system dependent and can be modeled on the procedures in the acceptance document provided by the manufacturer. Often, the tests can be taken directly from the acceptance document and performed in reasonable intervals, which should depend on the severity of the issue checked and on the complexity of the test.

All $\mathrm{kV}$ CBCT systems provide safety interlocks that are triggered by switches on the $\mathrm{kV}$ tube housing and/or imaging panel as well as the (robotic) arms holding these components. Depending on the system, once triggered an interlock will inhibit all motion of the entire linear accelerator system until a reset button is pressed or just until the interlock switch is released. The functionality of each interlock switch, also referred to as touch guard or collision detector, needs to be tested in regular intervals. Testing should involve attempts to move the couch or gantry while the interlock is engaged and verifying that all motion is stopped.

System interlocks that prevent operation of the $\mathrm{kV}$ imaging system under undesirable conditions (door open, $\mathrm{kV}$ source arm not fully extended) should also be tested by creating these conditions and verifying that the operation ceases immediately or not begin. The same applies to any "Terminate" or "Emergency off" key. Such key is tested by pressing it while Xrays are being emitted.

\subsection{Geometric accuracy}

This group of tests assesses how well the isocenter of the CBCT imaging system agrees with the isocenter of the MV treatment beam of the machine. Correct alignment of the patient 
depends directly on this agreement, and therefore these tests are very important and need to be performed daily. Accuracy levels are described in the literature (Klein et al., 2009). However, precise tests $(\sim 1 \mathrm{~mm})$ of the agreement of $\mathrm{kV} \mathrm{CBCT}$ and MV treatment isocenters are very comprehensive and time consuming. Therefore multiple levels of tests are generally used: faster and less precise ones for daily checks by Radiation Therapists and more precise tests for monthly checks and checks following system repair or upgrade by the Medical Physicist.

All tests have in common that a structure, often a sphere of some type, is imaged with the CBCT system and alignment with the MV treatment beam is checked. In the less involved tests, which will be called Level 1 in the following, the cross hair of the machine or even the room lasers are used to describe isocenter of the MV treatment beam. In the precise tests (Level 2) the treatment beam is engaged from multiple angles to describe its isocenter. The methodology of these tests goes back to Winston and Lutz (Lutz et al., 1988).

Current CBCT systems offer multiple imaging geometries (modes) with varying position of imaging panel and $\mathrm{kV}$ aperture to accommodate different patient sizes and treatment locations. If each of these modes has a separate calibration file within the $\mathrm{kV}$ imaging system, as is the case for the current Varian OBI and Elekta XVI systems, then it needs to be checked separately for isocenter accuracy, provided the mode is used clinically. This also applies to the direction of the gantry rotation during imaging. For a current Varian OBI system (including True Beam), accuracy tests would need to be performed for four scan types: (1) body scan, clockwise, (2) body scan, counter clockwise, (3) head scan, clockwise, and (4) head scan, counter clockwise. Similarly for the Elekta XVI system with the small, medium and large field of view, six accuracy tests would be needed, if all modes are used clinically. To keep the workload reasonable, these tests can be performed on a rotating schedule. Specific suggestions are made later in this chapter.

\subsubsection{Level 1 geometric accuracy test}

For daily checks the $\mathrm{kV} \mathrm{CBCT}$ isocenter should be compared to the cross hair of the machine as a representation of the MV beam isocenter. It is also possible to use the room lasers instead of the cross hair with the understanding that this introduces an additional uncertainty, which depends on the accuracy of the lasers.

The test requires a phantom that contains a structure, which can be well imaged with $\mathrm{kV}$ $\mathrm{CBCT}$ and also aligned visually with the cross hair. In a simple approach, one or more patient markers (BB) could be fixed on plate (Lehmann et al., 2007), as shown in figure 2. However, plastic markers should be used instead of the metal ones seen in the image, since they will lead to an improved CBCT image quality. Metal markers often cause streak artifacts in the CBCT image due to the metal - air and metal - plastic interfaces, which make alignment challenging.

A well suitable phantom is the Penta Guide phantom (Modus Medical system, London, ON, Canada). The phantom is shown in figure 3.

It is made from a plastic material and contains a spherical air-filled cavity in its center. It also contains additional spherical air-filled cavities. The location of the center air cavity is aligned with cross hair markers on the outside of the cube. The cross hair markers feature 


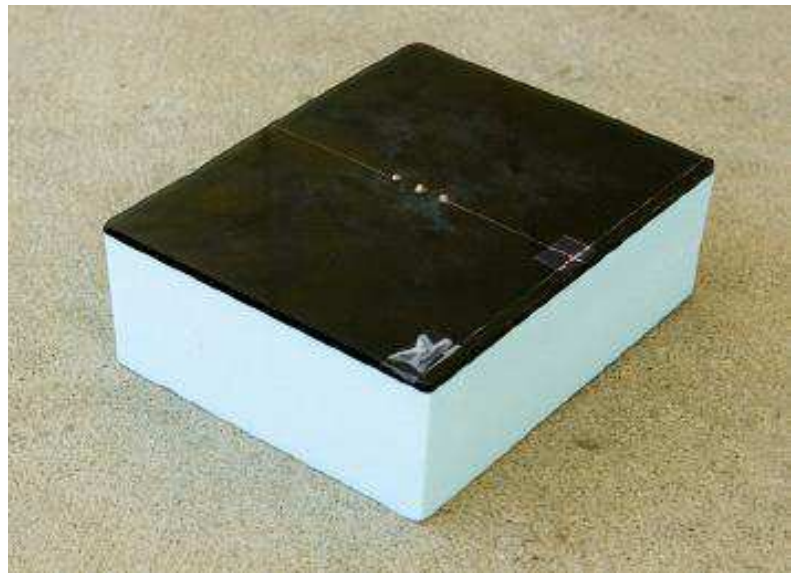

Fig. 2. Simple Level 1 geometry accuracy test phantom. Consisting of three small steel balls (BBs) mounted half-sunken in a Lucite plate on a Styrofoam base, this phantom is used verify agreement of the isocenters of the kilovoltage and megavoltage beams.

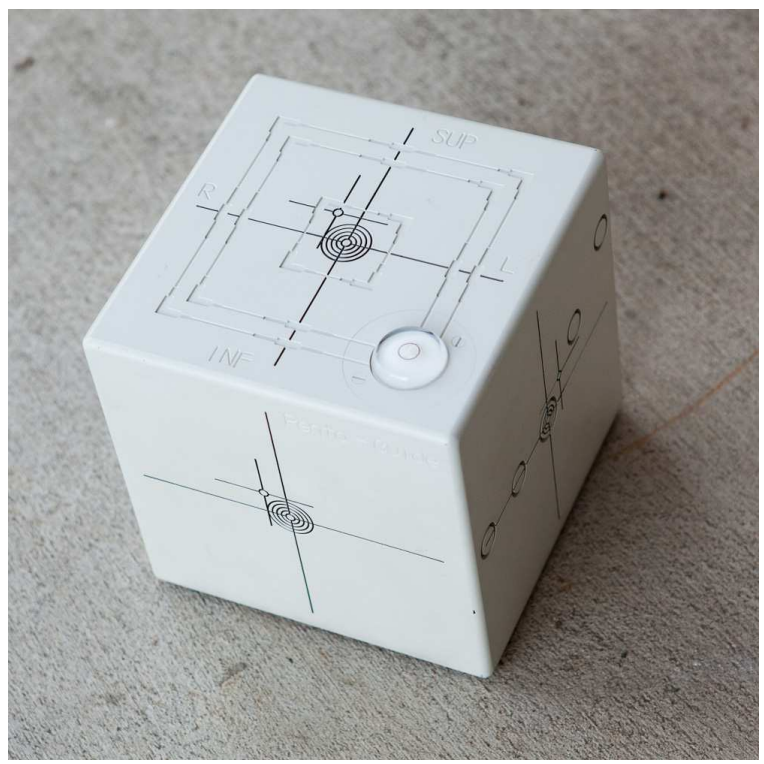

Fig. 3. Penta Guide phantom (Modus Medical, London, ON, Canada)

concentric circles, which allow for easy quantification of deviation. The phantom also has a second set of cross hair markers, which are correspond to a location $1 \mathrm{~cm}$ right, $1.2 \mathrm{~cm}$ up, $1.4 \mathrm{~cm}$ towards gantry with respect to the center of the cube. These can be used for a test of the position correction as described in section 2.3.

Another phantom is the "ISO Cube" (CIRS Inc, Norfolk, VA, USA). It is made from a plastic material and contains a unique center point fiducial as well as an offset blind target. The 
phantom measures $12 \mathrm{~cm} \times 12 \mathrm{~cm} \times 12 \mathrm{~cm}$, which offers advantages for systems with a shorter length of scanned volume.

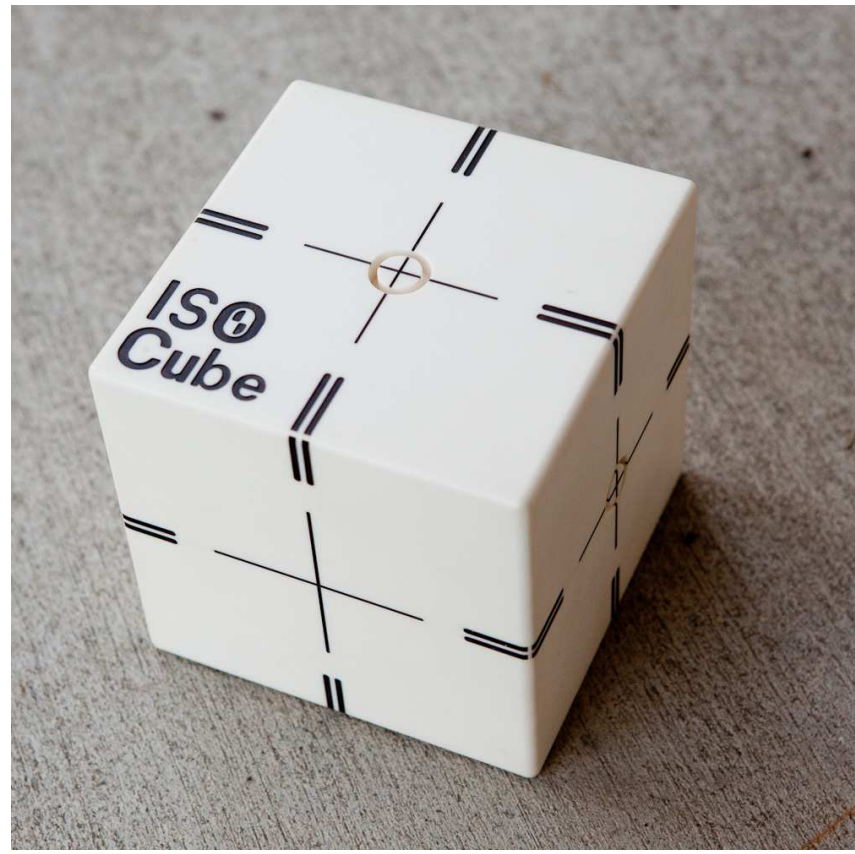

Fig. 4. ISO Cube (CIRS Inc, Norfolk, VA, USA)

To perform a Level 1 Geometric Accuracy Test for CBCT, the phantom is first lined up manually with the MV isocenter using the field cross hair at two gantry angles: $0^{\circ}$ and $90^{\circ}$ (or $270^{\circ}$ ) IEC scale. Then a CBCT imaging procedure is performed. Agreement of the center of the phantom in the CBCT software is checked against the center of the image, as marked in the system. In the simplest case any reference image data set can be used and agreement of the location of the center of the center sphere is checked against the marked isocenter of the system. For a routine procedure it is recommended to use a CT image of the phantom as reference data set. In order to create such reference data set, the phantom is scanned with a conventional CT scanner using a thin slice thickness of 1 or $1.5 \mathrm{~mm}$. The CT data set is then sent to the treatment planning system, where the isocenter is carefully located and marked. A treatment beam is assigned to the isocenter. For some systems it is also necessary to create at least one structure. If applicable, a test patient is created in the record and verify system and the reference image set including structure and beam data is sent there, just like a the information of a patient would be.

With these preparatory steps the Level 1 Geometric Accuracy Test can now be performed using a reference image of the phantom and aligning the CBCT to it. For obvious reasons it is crucial to have the isocenter marked correctly in the reference data set.

One could also perform the test in reverse order, which is to first align the phantom using $\mathrm{CBCT}$ to the isocenter of the $\mathrm{CBCT}$ system and then check the alignment against the MV 
system using cross hair (or laser). While conceptually more aligned with the clinical process of patient setup, a drawback of this approach is the limited resolution (and accuracy) of the couch movement of many current systems $(1 \mathrm{~mm})$, which limits the accuracy with which the phantom can be aligned with the $\mathrm{kV} \mathrm{CBCT}$.

Still, the latter order is often used, as it allows for easy combination with the check of the registration accuracy as described in section 2.3.

\subsubsection{Level 2 geometric accuracy test}

For a more precise check of the agreement of the $\mathrm{kV} \mathrm{CBCT}$ isocenter with the MV beam isocenter, the MV beam isocenter is described with MV imaging at multiple gantry and possibly collimator angles.

This test requires a phantom that can be imaged well with $\mathrm{kV} \mathrm{CBCT}$ and also with MV imaging. The Penta Guide (Figure 3) offers limited image quality for MV imaging. However, the authors have used it successfully over several years. The ISO Cube phantom (Figure 4) offers better MV imaging, but has less favorable $\mathrm{kV}$ CBCT image quality. A phantom that offers very good $\mathrm{kV} \mathrm{CBCT}$ and MV image quality has been adopted by the authors from Greg Gibbs and Jerry White of the Colorado Associates in Medical Physics. The phantom consists of a platform with a small tube upright mounted on it. The tube can hold one of two identically sized balls (diameter $\sim 8 \mathrm{~mm}$ ). For $\mathrm{kV} \mathrm{CBCT}$ a plastic (Lucite) ball is placed on the tube and for MV imaging a Tungsten ball. The phantom, which has been called Two Ball Phantom (TBP), is shown in figures 5 and 6.

The procedure for the level 2 test is analogous to the one for level 1. The phantom is setup to one modality, in this case the $\mathrm{kV} \mathrm{CBCT}$, as it allows for better adjustments of the position. The before mentioned problem of the limited precision of the table movement can be eased by recording the final deviation from which no more moves are possible (due to precision limit) in each dimension and comparing it later with the deviation seen with the MV imaging.

If the precision of the on-screen display is also only $1 \mathrm{~mm}$, which is common and reasonable for clinical applications, the deviation can be estimated by first aligning the images as good as possible on the screen and then making discrete steps (using the keyboard) in each direction until the next digit turns. This needs to be done both ways to determine how many "clicks" equal $1 \mathrm{~mm}$.

After best alignment with $\mathrm{kV} \mathrm{CBCT}$ has been achieved and additional deviations are noted as described, the phantom is imaged with the MV beam at gantry angles $0^{\circ}, 90^{\circ}, 180^{\circ}$, and $270^{\circ}$. This can be done using film or the portal imager. Film measurements will require precise alignment of the film perpendicular to the beam and subsequent scanning for best results. The use of portal imaging is recommended and will be described in the following. However, the method can also be applied to film.

To reduce errors in the position of the portal imager, a fixed jaw or MLC geometry is used and the position of the image of the phantom is evaluated relative to the so described radiation field. To reduce errors due to small inaccuracies in jaw or MLC position the images are taken at two different collimator angles (preferably $90^{\circ}$ and $270^{\circ}$ ) for each gantry angle. 


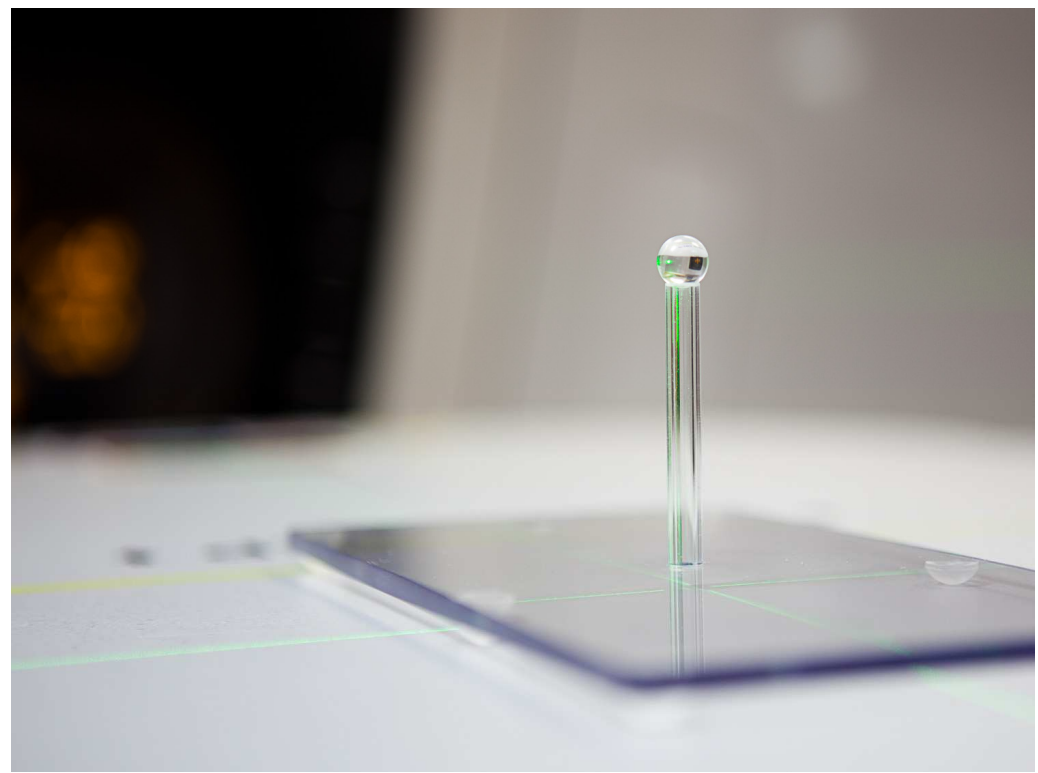

Fig. 5. Two Ball Phantom: A platform with a small tube as a riser holds one of two same-size balls for imaging, a plastic ball shown here for $\mathrm{kV} \mathrm{CBCT}$ and a Tungsten ball for MV imaging as shown in figure 6.

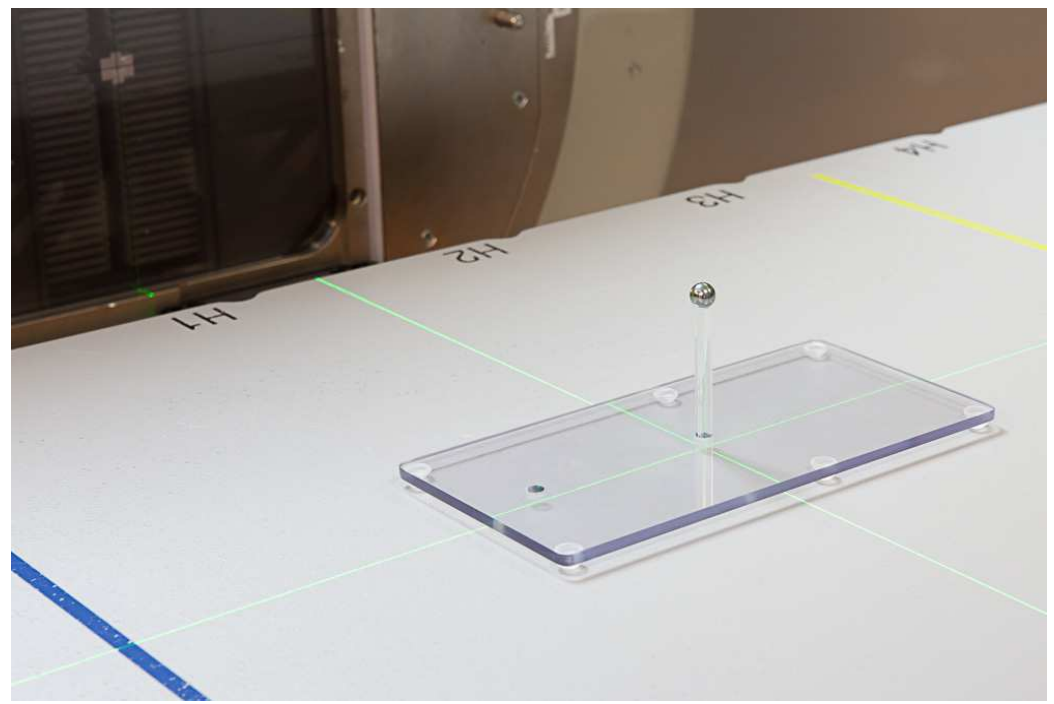

Fig. 6. Two Ball Phantom setup with Tungsten ball for MV imaging and small MLC shaped field.

On each image the position of the ball relative to the field edges is analyzed. This can be done visually for a first and approximate confirmation. Image analysis tools of the MV 
portal imaging software or another software can be used to assist in manual analysis. Figure 7 shows an example of the center of the Penta Guide phantom being imaged.

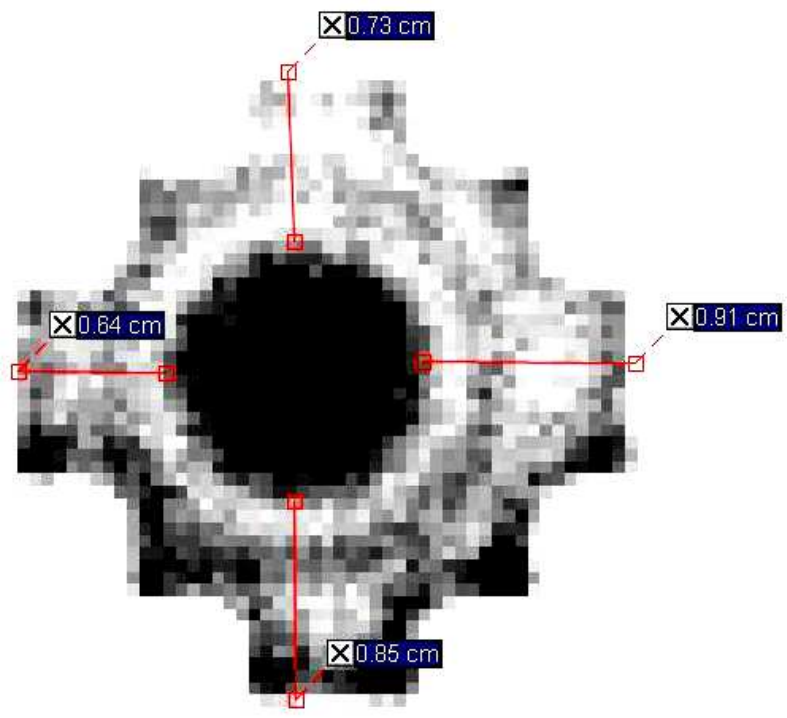

Fig. 7. MV image of the central sphere in the Penta Guide phantom with a small MLC shaped field. The measurement tool in the OBI software has been used to estimate the position of the image of the sphere relative to the field. Note: The poor image quality is (mainly) the result of the underlying physics, not of the reproduction.

Figures 8 shows $\mathrm{kV}$ and MV images of the plastic and Tungsten ball of the TBP. Clearly the MV image quality of the TBP is superior to the one of the Penta Guide. Both MV images also show the lines from the measurement tool that was used to assess the distance of the edge of the cavity (Penta Guide) or ball (TBP) from the edge of the field.

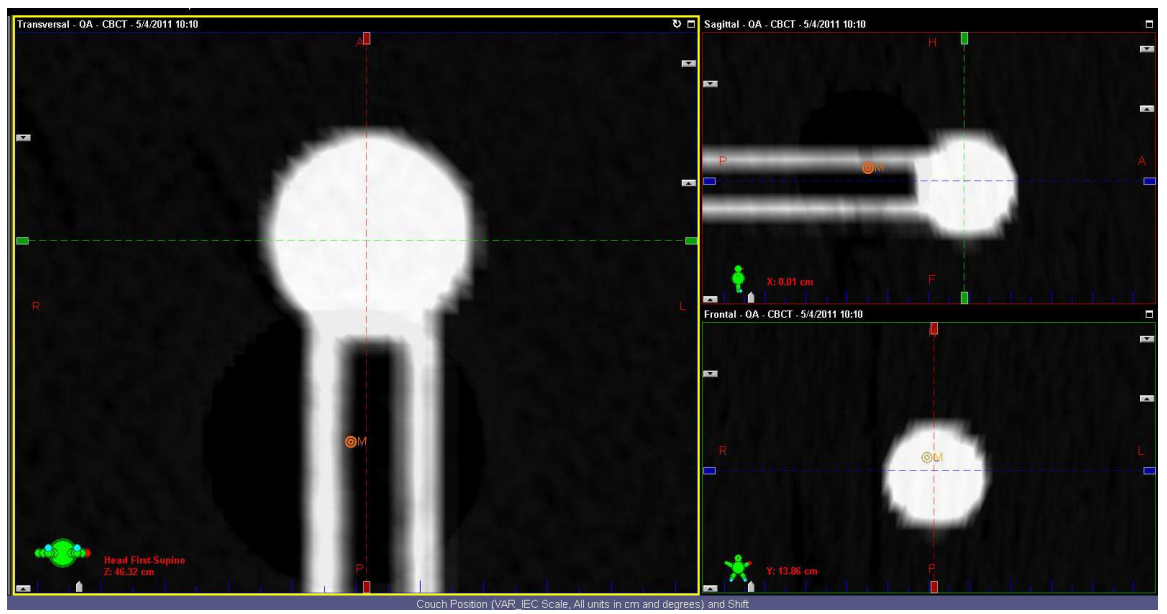




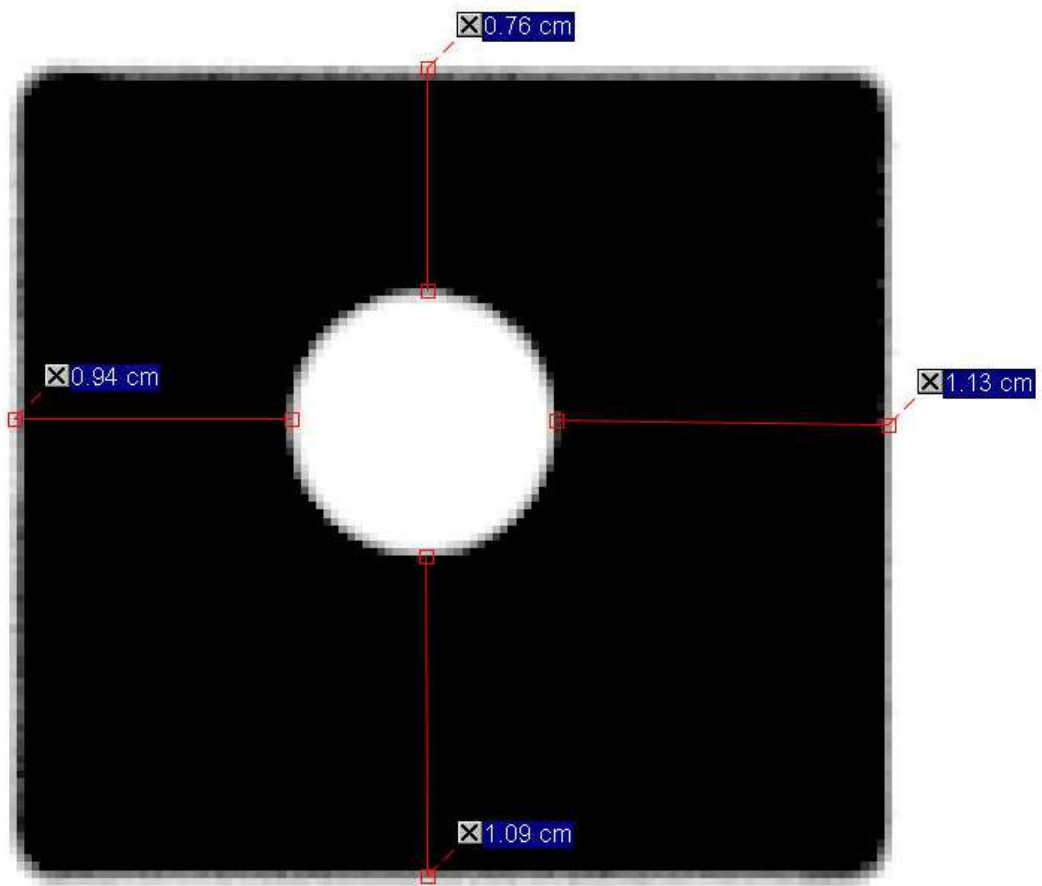

Fig. 8. Two Ball Phantom, top: kV CBCT image of the plastic ball in three views, bottom: MV port film image of the Tungsten ball (Varian OBI).

\subsection{Registration and correction accuracy}

In addition to checking the accuracy of the isocenter of the imaging system, it needs to be verified that the system calculates shifts of a slightly misaligned patient's planned isocenter to the machine isocenter correctly. It should also be verified that these shift are executed correctly (couch movement), if this feature is used clinically. If this process could not be trusted, a confirmation CBCT of the patient in the final position would need to be taken after each shift to verify that patient treatment and machine isocenter agree following the shift. The magnitude of the shifts to be checked should be as large as the largest magnitude expected in clinical use.

This test uses the same phantom as the isocenter accuracy test and is best done with a prepared reference CT scan of the phantom as described in section 2.2 above. The phantom is setup to an off center location. It is then CBCT imaged and the shifts are determined with the CBCT software. Some phantoms, such as the Penta Guide offer a second setup point with known shifts from the center. If that is used the values of the determined shifts can be compared to the expected values. Otherwise, or in general for a more thorough QA (monthly), the shifts are applied (couch moved), a second CBCT is performed and the alignment of the phantom is verified in the CBCT software. If the system functions properly, only very small shifts within the accuracy of the couch movement should now be needed for alignment of CBCT with the reference image. 
Following the alignment of the images in the software, the physical position of the phantom can be verified with respect to the machine isocenter using the cross hair of the accelerator. This effectively combines this registration and correction accuracy check with the isocenter accuracy check described in section 2.2. However, the combined test is potentially limited by the accuracy of the couch motion.

\subsection{Image quality}

Image quality needs to be measured in regular intervals to ensure stability of the imaging chain and to guarantee the lowest imaging dose to the patient. A suitable tool is the Catphan (The Phantom Lab, Salem, NY), as shown in figure 9. The phantom consists of a housing containing several modules with geometrical structures to test variousimage quality parameters.

Currently, both manufacturers of $\mathrm{kV} \mathrm{CBCT}$ systems provide a version of the phantom with the CBCT system. The Catphan phantom is setup on the treatment couch of the linac system. It hangs from its wooden box over the end of the couch, so that no part of the couch is between phantom and image source or panel. For consistency it is important to keep the phantom leveled. (Figure 10)

The phantom should be setup with the machine isocenter at approximately its center (vertical laser between second and third module). With this approach all modules of the phantom can be captured in one CBCT image set.

There is another approach, which is currently used in the acceptance testing of the Elekta XVI system. Here separate scans are performed for the separate modules of the phantom. A smaller field size is used in longitudinal direction. The phantom is setup to each module individually with the machine isocenter placed at the center of the particular module. This will potentially lead to better results, as in higher measured parameters. However, since the objective of the ongoing QA is to monitor changes in performance, imaging the entire phantom at once seems more suitable with regard to workflow.

Following the scan(s) of the Catphan, each module is analyzed. This is done best according to the procedure laid out in the acceptance document of the machine or using an independent software.

It can be argued that the spatial resolution test is most important, since it can detect changes to the focal spot of the x-ray tube. The test uses line pairs that are visually analyzed, as can be seen in figure 11. Visual analysis is subjective and can vary with the environment. However, it is widely used. The phantom section with the line pairs offers also a small element that can be used for automatic analysis. The image of a thin wire (small white dot towards the center of the image in figure 11) can be analyzed with suitable software to determine to modulation transfer function of the system.

Another very important set of tests are the geometric tests, which test parameters of the imaging geometry: Using measurement tools of the software or a third party application, it is determined how accurate known distances in the phantom are represented in the image (Figure 12). This is important since shifts of the patient following the CBCT imaging will be determined based on distances in the image. 


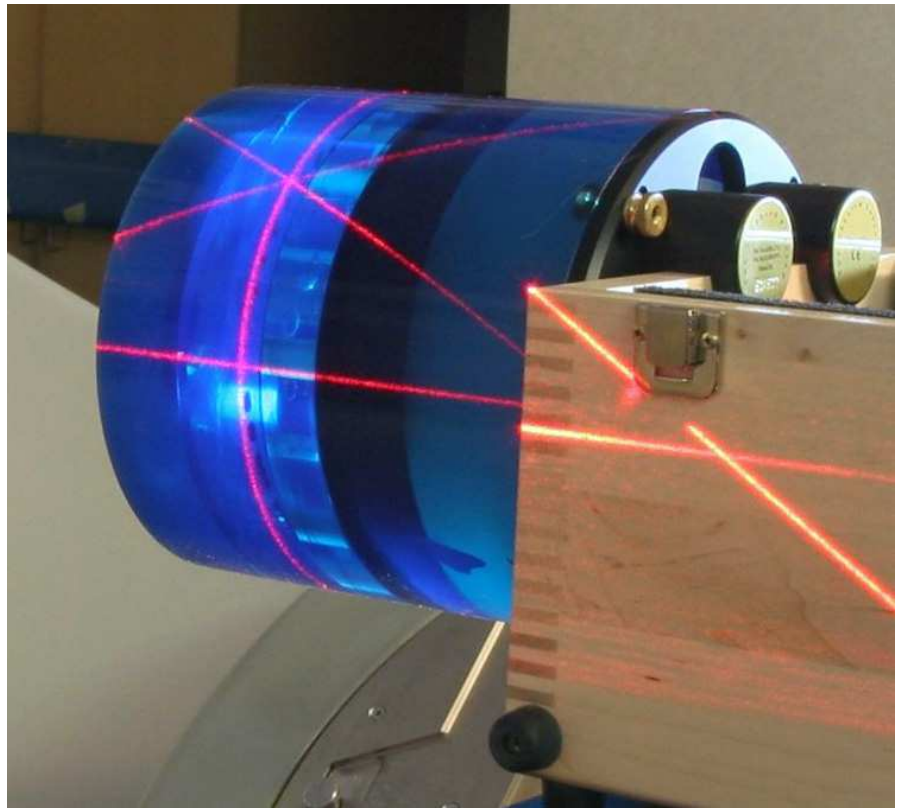

Fig. 9. Catphan: Phantom for image quality tests.

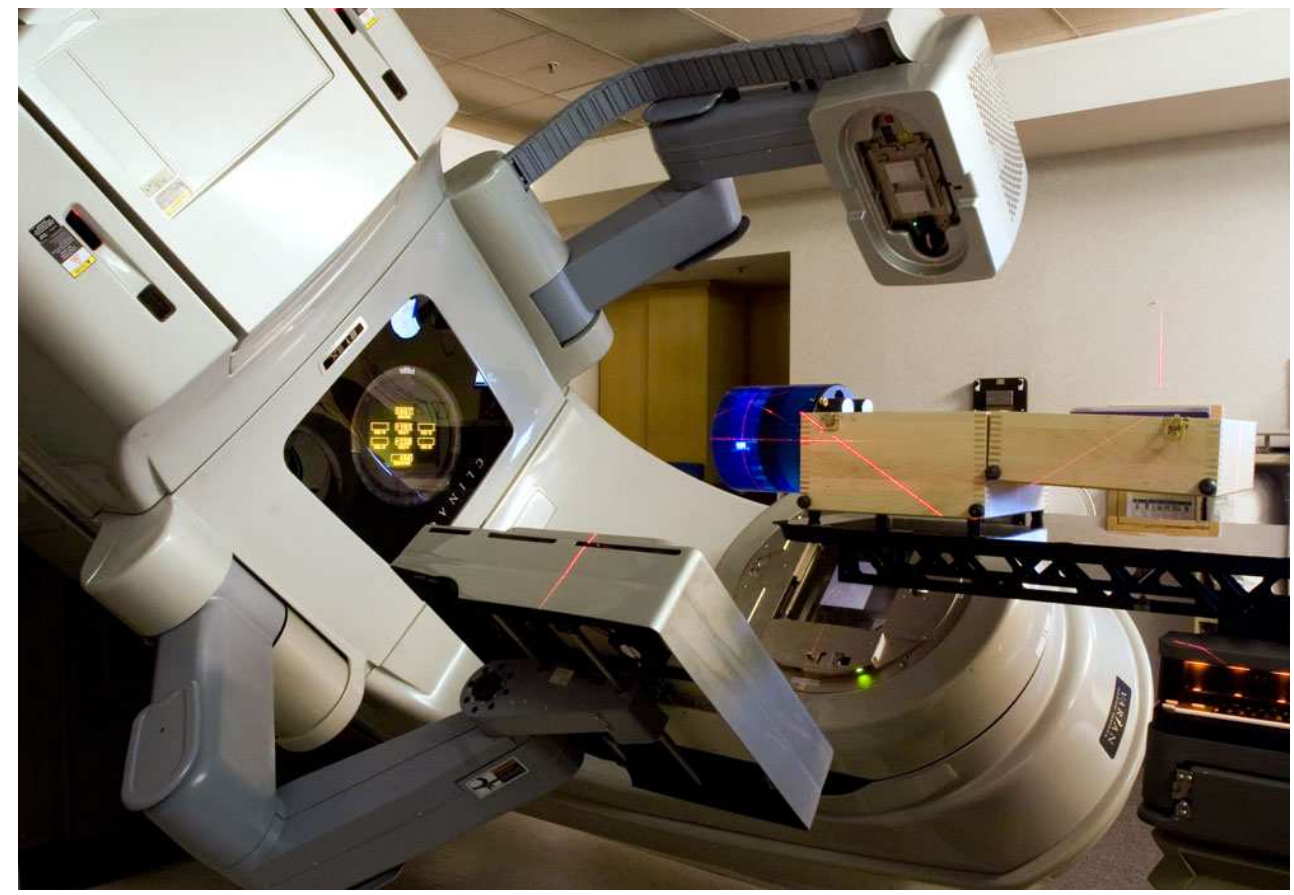

Fig. 10. Setup of Catphan at machine Isocenter to be imaged for image quality tests. 


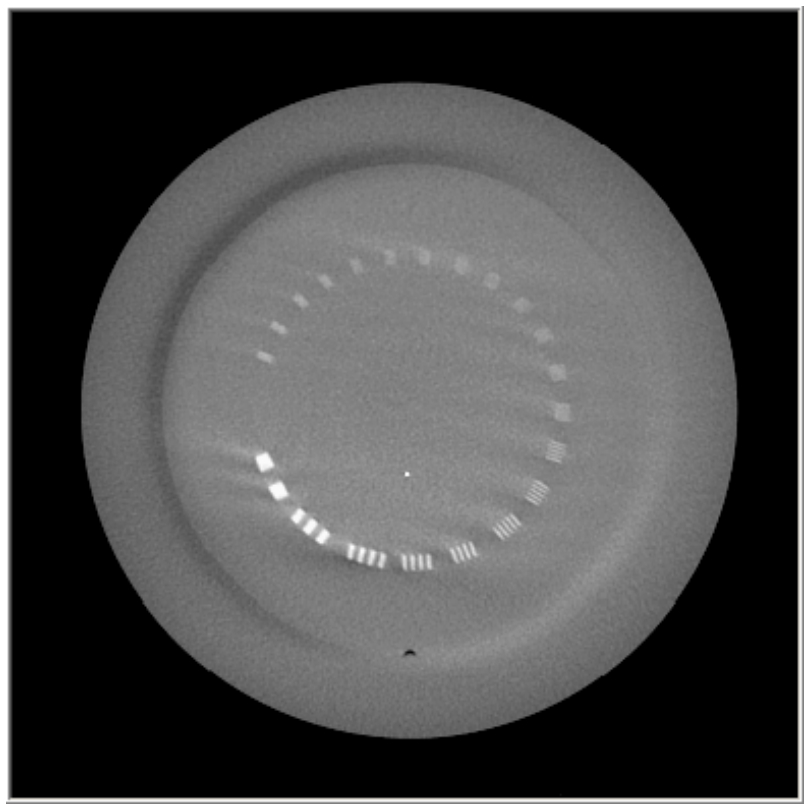

Fig. 11. CBCT image of a slice of the Catphan phantom used to determine the spatial resolution of the imaging system.

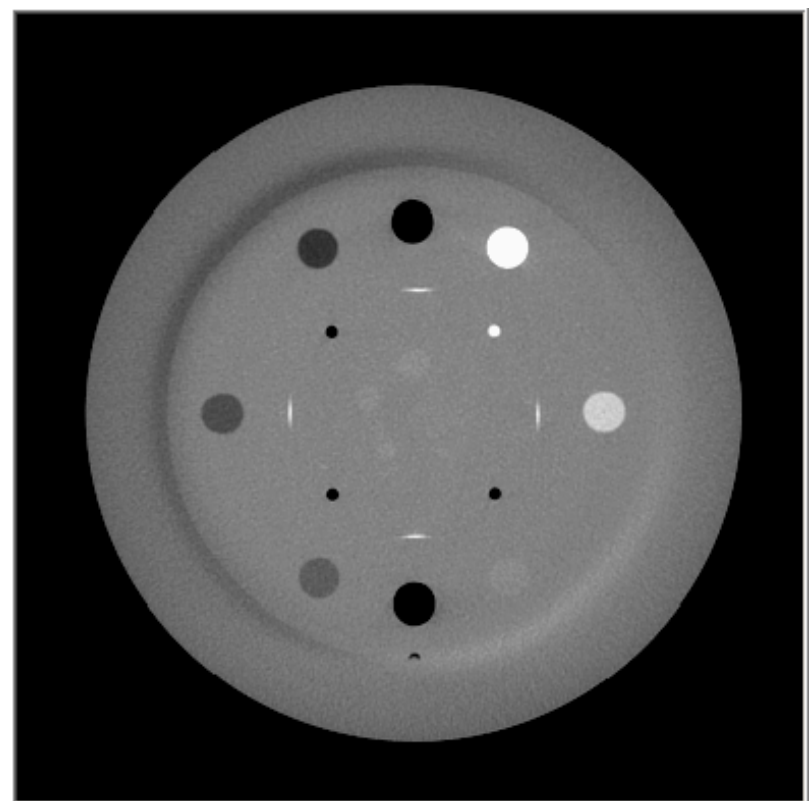

Fig. 12. CBCT image of a slice of the Catphan phantom with samples of various materials of known d density (outer ring of circles) as well as with precise geometrical markers. 
CT number linearity can also be checked with the Catphan. The module that is used for the geometry tests also provides samples of various materials with known densities (Figure 12). The Acceptance Test of the Elekta XVI system uses these CT numbers to calculate low contrast visibility. Markers in this module can be used to calculate slice thickness, which is, however, less relevant for CBCT.

Low contrast visibility can also be determined with another module (Figure 13) and is currently done so for the Varian Acceptance testing. This test depends on the user being able to see circles of different diameters in the image.

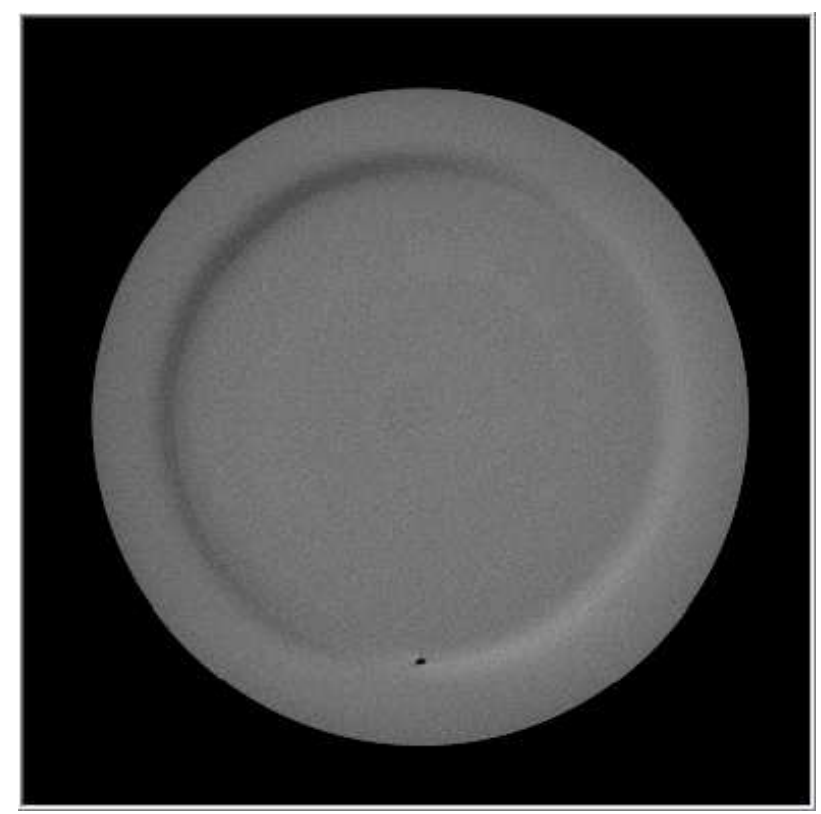

Fig. 13. CBCT image of a slice of the Catphan phantom used to determine Low Contrast Visibility.

Lastly, there is a uniformity check, which compares the CT number in different parts of the image of a homogenous phantom. This test can be done easily by hand with the tools in the imaging software.

For all image quality checks consistency of the check execution is more important than how exactly the test is done. The image quality tests should be done with a parameter set that produces a high image quality. The tests do not have to be repeated with all clinically used imaging parameters, as any significant changes in the system would be visible in test with the high quality.

All tests can be done with the tools available on the imaging software. However, user independent tests using software to analyze the images give more objective measures of the stability of the system. Several vendors have products that allow user independent analysis and also varying degree of automation of the QA. Companies with such products are 
Standard Imaging (Middleton, Wisconsin, USA) and Radiological Imaging Technology (Colorado Springs, Colorado, USA)

\subsection{X-ray tube and generator tests}

The CBCT imaging chain consists of the $x$-ray generator, $x$-ray tube, and the digital imaging device. It is important to assure that the generator and $x$-ray tube are performing properly in a known manner so that imaging technique parameters can be confidently adjusted by the user. For example, if the user wishes a less noisy image, he should be confident that increasing the $\mathrm{mA}$ setting by $50 \%$ results in $50 \%$ more radiation being incident on the patient.

A fairly comprehensive set of measurements should be performed at time of acceptance. A more streamlined set should be performed periodically thereafter and following major component changes. The desired data are easily taken with a number of commercially available diagnostic imaging products, e.g. by Unfors (Billdal, Sweden) and RTI Electronics AB (Mölndal, Sweden) that provide $\mu \mathrm{Gy}$, exposure time, and HVL in a single measurement. These tests do require that the CBCT be operated in a radiographic, as opposed to CT, mode. The way to operate in this manner varies with vendor.

The tests to be performed and parameters to be measured are the same for the baseline acceptance tests and the periodic tests. The latter is a subset of the former and concentrates on those settings used for clinical CBCT protocols. The tests should be performed in a readily reproduced geometry that allows the measurement device to obtain accurate readings. Since most of the devices require a certain minimum amount of radiation for a reading, it may be necessary to set the detector some 60 to $80 \mathrm{~cm}$ from the $x$-ray source. This is easily accomplished through of simple platforms made of readily available polystyrene blocks.

The required tests fall into three general categories:

1. $\mathrm{kVp}$ accuracy and HVL: Measure the $\mathrm{kVp}$ and compare to the value chosen on the generator. Also record the $\mu \mathrm{Gy}$ at these setting so that the $\mu \mathrm{Gy} / \mathrm{mAs}$ performance can be recorded for future reference. Perform these measurements for the full range $\mathrm{kVp}$ settings and for both large and small focal spots. Record the HVL at a clinically used $\mathrm{kVp}$ such as $120 \mathrm{kVp}$. Note that the measured HVL for a CT tube is substantially higher than a conventional diagnostic $x$-ray tube.

2. Timer accuracy and linearity: At a fixed, clinically used $\mathrm{kVp}$, measure the exposure time and $\mu \mathrm{Gy}$ for a full range of allowed timer setting. Timer values should agree with the set time readings, while the $\mu \mathrm{Gy} / \mathrm{mAs}$ should remain constant.

3. $\mathrm{mA}$ linearity: It is difficult to measure tube $\mathrm{mA}$ directly in a non-invasive fashion. The true $\mathrm{mA}$ is less important than the output, $(\mu \mathrm{Gy})$, tracking in a linear fashion with the set $\mathrm{mA}$. Measure the $\mu \mathrm{Gy}$ for the full range of allowed $\mathrm{mA}$ settings at fixed, clinically used $\mathrm{kVp}$, for both small and large focal spots. Compute the $\mu \mathrm{Gy} / \mathrm{mAs}$ by dividing the $\mu \mathrm{Gy}$ by set $\mathrm{mAs}$. This value should be approximately constant over the full range of $\mathrm{mA}$ settings and between focal spots.

A comprehensive baseline should be obtained at time of acceptance. Acceptable performance occurs when the measured value is within $10 \%$ of the set value $(\mathrm{kVp}, \mathrm{mA})$ or 
measured mean value ( $\mu \mathrm{Gy} / \mathrm{mAs})$. Any unacceptable results should be resolved with the vendor. Ongoing checks should be performed on an annual basis or following a change in major system component, e.g. the $\mathrm{x}$-ray tube. The annual test can be limited to those $\mathrm{kVp}$ settings used for clinical protocols and to a limited number of $\mathrm{mA}$ and time settings that encompass the range used in the clinical protocols.

\subsection{End-to-end test}

As for all systems that help position the patient prior to the application of radiation therapy, the ultimate verification is an end-to-end test, in which a phantom is put through the entire chain of procedures that the patient goes through and where the received radiation dose in the phantom can be recorded and visualized.

Radiochromic film, which is relatively insensitive to visible light but changes its color proportional to the amount of ionizing radiation received, is an elegant tool for end-to-tests. This also applies to $\mathrm{kV} \mathrm{CBCT}$ systems since the $\mathrm{kV}$ radiation involved in CBCT imaging does not have a visible effect on the film. For straightforward, visual verification an end-toend test is planned with an arc field as the treatment field to be delivered to the phantom. An arc, which covers close to 360 degrees, produces a circular darkening of the film around the isocenter when the film is placed perpendicular to the couch (Figure 14). Using a precisely machined hole, which meets the film at center of the phantom, a needle can be used to mark the center of the phantom on the film.

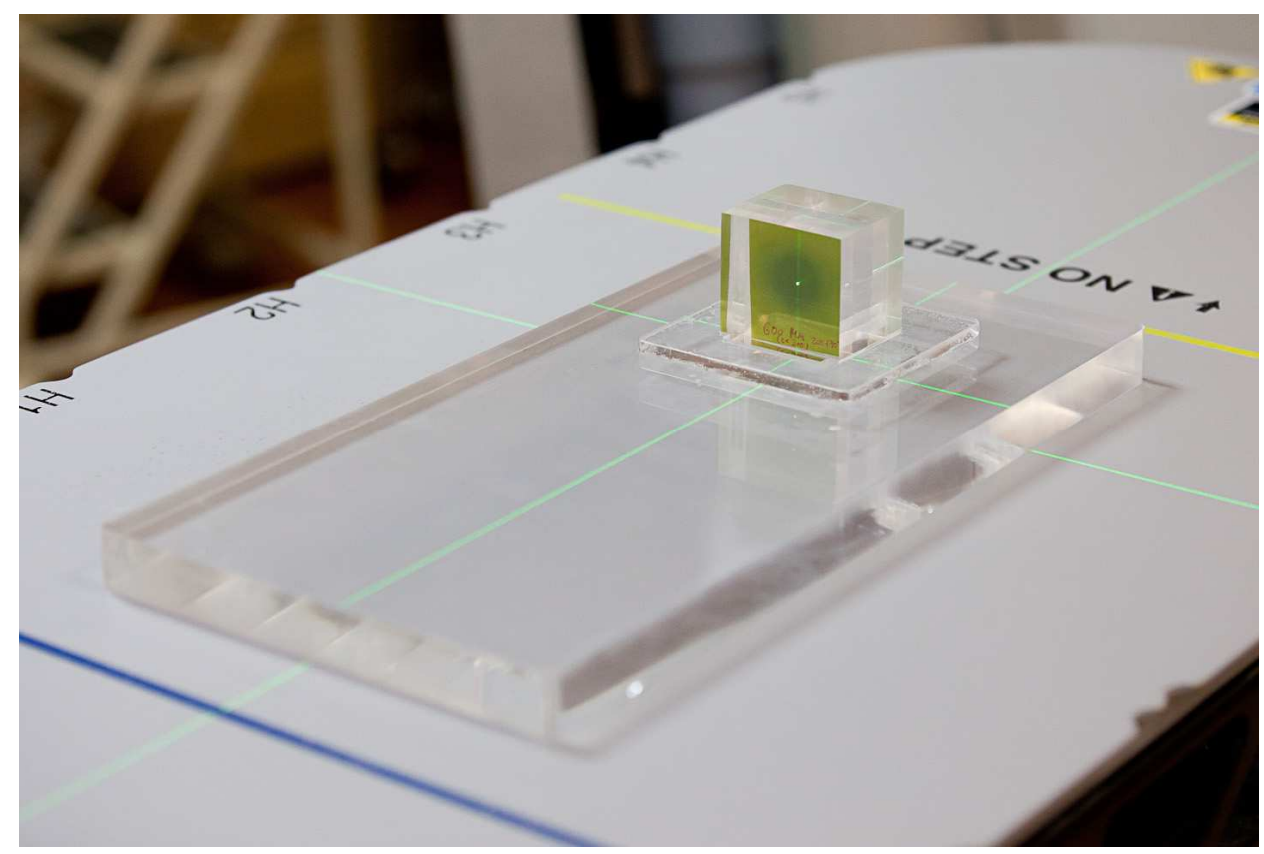

Fig. 14. Phantom for and end-to-end test with radiochromic film after an arc of therapeutic radiation has been delivered to it. 
Using CBCT, the phantom is setup to align its center with the isocenter of the beam. After the arc field has been delivered, the relationship between the needle poke and the circular darkening can be evaluated to determine the agreement of the alignment in anteriorposterior and left-right direction. Visual evaluation can be supplemented with softwarebased analysis of the film.

To assess the isocenter agreement in superior-inferior direction, the film needs to be rotated by $90^{\circ}$ around the anterior-posterior axis. The shown phantom is build to accommodate precise rotation of the block with the film (Figure 15) by $90^{\circ}$, while maintaining isocenter position. Alternatively a separate test can be performed with the entire phantom rotated.
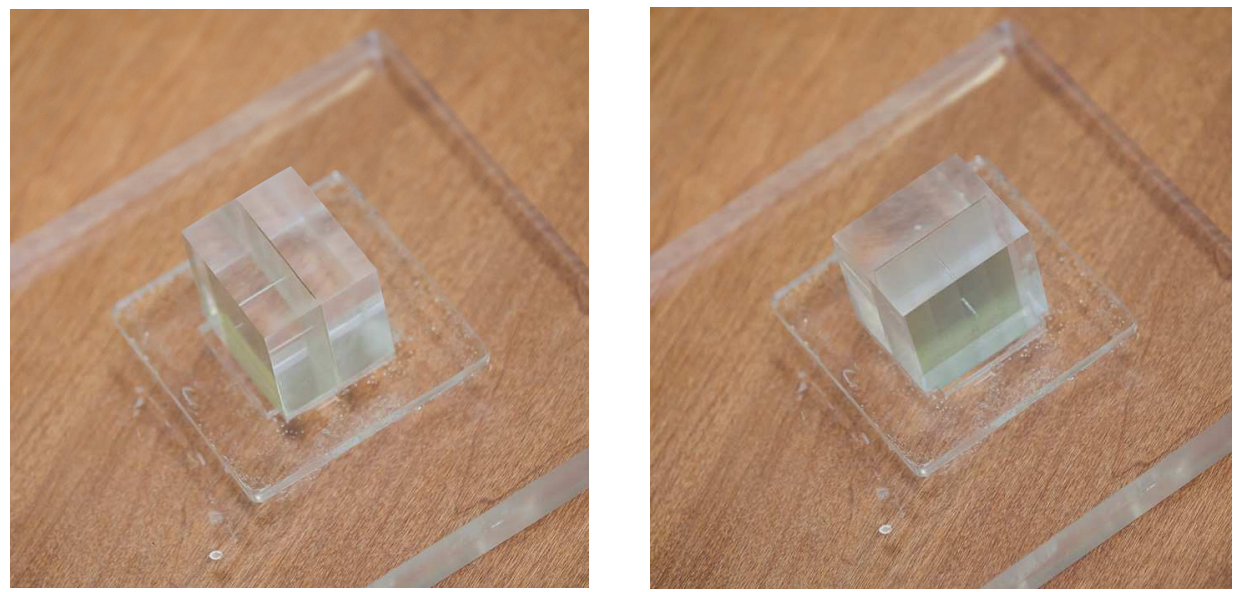

Fig. 15. Phantom for and end-to-end test using a plastic cube as holder for radiochromic film, which can be rotated precisely by $90^{\circ}$ while maintaining isocenter.

\section{Frequency of tests}

\subsection{Daily QA}

Safety checks: Depending on the specifics of the system the available interlocks should be either all tested, or tested on a rotational basis throughout the week.

Level 1 Geometric Accuracy Test: For daily checks the $\mathrm{kV} \mathrm{CBCT}$ isocenter should be compared to the cross hair of the machine.

Registration and correction accuracy: A phantom should be manually setup to an off-center location. The phantom should be CBCT imaged, aligned and moved to isocenter. This test can be combined with the geometric accuracy test, if the final location of the phantom is compared to the cross hair of the beam or to the in-room laser system, provided that has been checked.

\subsection{Monthly QA}

Safety checks: All available interlocks should be tested monthly 
Level 2 Geometric Accuracy Test: The isocenter of the kV CBCT system should be compared to the isocenter of the MV system as represented by a portal image with the treatment beam.

Registration and correction accuracy: The daily QA of the Registration and correction accuracy should be repeated by the physicist performing the monthly QA.

Image quality check: The described image quality checks with the Catphan should be performed. Preferably all tests should be done each month.

\subsection{Annual QA}

X-ray Tube and Generator Tests should be performed annually, as described above. An endto-end test should also be performed.

\subsection{QA following system repairs or upgrades}

Following a system repair or upgrade that did not involve the x-ray tube and/or generator the tests of the monthly QA should be performed.

If $\mathrm{x}$-ray generator and/or generator were involved in the intervention, an annual QA should be done in addition to the tests of the monthly QA.

Depending on the severity of the repair or upgrade, an end-to-end test should also be performed. If the repair or upgrade on affected the CBCT system but not the first part of the chain (CT scanner, treatment planning system, record and verify system), only the final step of the end-to-end test needs to be done.

\section{Summary}

Image guided therapy in general and kV Cone Beam Computed Tomography in particular are powerful tools in a modern Radiation Oncology facility. Assuring the correct function of all systems is of utmost importance in the interest of accurate patient treatment.

The described tests, which should be performed or supervised by a Qualified Medical Physicist, help assure a high level of patient care. For additional information refer to current literature, such as (Bissonnette 2007) and (Bissonnette et al., 2008), and to upcoming publications from professional organizations, such as the AAPM Task Group on CBCT QA.

\section{List of abbreviation and acronyms}

AAPM - American Association of Physicists in Medicine

CBCT - Cone Beam Computed Tomography

CT - Computed Tomography

Gy - Gray - Unit for the absorbed radiation dose in medium (1 Gray = 1 Joule / 1 Kilogram)

HVL - Half Value Layer

IGRT - Image Guided Radiation Therapy

$\mathrm{kV}$ - Kilo voltage

$\mathrm{kVp}$ - Kilo voltage peak value (max $\mathrm{kV}$ in a beam with photons of a range of $\mathrm{kV}$ )

$\mathrm{mA}$ - Milliampere 
mAs - Milliampere Seconds

MLC - Multi Leaf Collimator (Beam shaping device of the linear accelerator)

MV - Mega voltage

OBI - On Board Imaging (product of Varian Medical Systems, Inc.)

QA - Quality Assurance

QMP - Qualified Medical Physicist

TBP - Two Ball Phantom

TG - Task Group (here: Task Group of the AAPM)

XVI - X-ray Volume Imaging (product of Elekta AB)

\section{References}

Bissonnette, J. P. “Quality assurance of image-guidance technologies.” Semin Radiat Oncol 17(4): 278-286, 2007.

Bissonnette, J. P., D. Moseley et al. "Quality assurance for the geometric accuracy of cone beam CT guidance in radiation therapy." Int J Radiat Oncol Biol Phys 71(1 Suppl):S57-S61., 2008

Jaffray, D. A., D. G. Drake et al. "A radiographic and tomographic imaging system integrated into a medical linear accelerator for localization of bone and soft-tissue targets." Int J Radiat Oncol Biol Phys 45(3): 773-789. 1999

Klein, Eric E., Joseph Hanley, John Bayouth et al "Task Group 142 report: Quality assurance of medical accelerators" Med. Phys. 36 (9), 2009

Lehmann Joerg, Julian Perks, Sheldon Semon, Rick Harse, and James A. Purdy, "Commissioning experience with cone-beam computed tomography for imageguided radiation therapy" JACMP 8(3), 2007

Lutz W, Winston KR, Maleki N. "A system for stereotactic radiosurgery with a linear accelerator" IJROBP 14(2):373-81. 1988

Yin, Fang-Fang, John Wong, et al "The Role of In-Room kV X-Ray Imaging for Patient Setup and Target Localization" Report of AAPM Task Group 104 ISBN: 978-1-888340-891,2009 


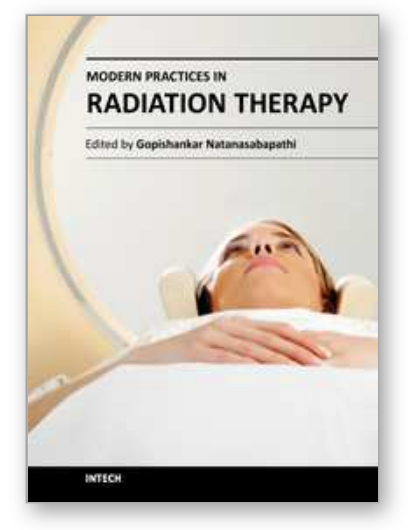

\author{
Modern Practices in Radiation Therapy \\ Edited by Dr. Gopishankar Natanasabapathi
}

ISBN 978-953-51-0427-8

Hard cover, 370 pages

Publisher InTech

Published online 30, March, 2012

Published in print edition March, 2012

Cancer is the leading cause of death in economically developed countries and the second leading cause of death in developing countries. It is an enormous global health encumbrance, growing at an alarming pace. Global statistics show that in 2030 alone, about 21.4 million new cancer cases and 13.2 million cancer deaths are expected to occur, simply due to the growth, aging of the population, adoption of new lifestyles and behaviors. Amongst the several modes of treatment for cancer available, Radiation treatment has a major impact due to technological advancement in recent times. This book discusses the pros and cons of this treatment modality. This book "Modern Practices in Radiation Therapy" has collaged topics contributed by top notch professionals and researchers all around the world.

\title{
How to reference
}

In order to correctly reference this scholarly work, feel free to copy and paste the following:

Joerg Lehmann and Stanley Skubic (2012). Quality Assurance (QA) for Kilovoltage Cone Beam Computed Tomography (CBCT), Modern Practices in Radiation Therapy, Dr. Gopishankar Natanasabapathi (Ed.), ISBN: 978-953-51-0427-8, InTech, Available from: http://www.intechopen.com/books/modern-practices-in-radiationtherapy/quality-assurance-for-cone-beam-computed-tomography-

\section{INTECH}

open science | open minds

\section{InTech Europe}

University Campus STeP Ri Slavka Krautzeka 83/A 51000 Rijeka, Croatia Phone: +385 (51) 770447 Fax: +385 (51) 686166 www.intechopen.com

\section{InTech China}

Unit 405, Office Block, Hotel Equatorial Shanghai

No.65, Yan An Road (West), Shanghai, 200040, China

中国上海市延安西路65号上海国际贵都大饭店办公楼405单元

Phone: +86-21-62489820

Fax: +86-21-62489821 
(C) 2012 The Author(s). Licensee IntechOpen. This is an open access article distributed under the terms of the Creative Commons Attribution 3.0 License, which permits unrestricted use, distribution, and reproduction in any medium, provided the original work is properly cited. 